JOURNAL OF

APPLIED

CRYSTALLOGRAPHY

ISSN 1600-5767

Received 9 February 2018

Accepted 14 March 2018

Edited by J. M. García-Ruiz, Instituto Andaluz de Ciencias de la Tierra, Granada, Spain

Dedicated to the memory of H.D. Megaw (1907-2002)

Keywords: handedness; chirality; quartz; optical rotation.

\section{Confusion over the description of the quartz structure yet again}

\author{
A. M. Glazer* \\ Physics Department, University of Oxford, Clarendon Laboratory, Parks Road, Oxford OX1 3PU, UK. *Correspondence \\ e-mail: glazer@physics.ox.ac.uk
}

In a recent paper [Huang, Gog, Kim, Kasman, Said, Casa, Wieczorek, Hönnicke \& Assoufid (2018). J. Appl. Cryst. 51, 140-147], a description of the structure of quartz was given that is incorrect. There is a long history of such errors in publications describing the quartz structure. This was fully and correctly discussed in 1978 [Donnay \& Le Page (1978). Acta Cryst. A34, 584-594], and yet these errors still persist. In the present paper the description by Huang et al. is corrected and the seminal work of Donnay and Le Page revisited.

\section{Introduction}

And should not I pity Nineveh, that great city, in which there are more than 120000 persons who do not know their right hand from their left, and also much cattle? (Jonah 4:11.)

Publications on the structure of quartz have a long history of errors and confusion. There are so many errors in the literature that several writers (Lang, 1965; Barron et al., 1976; Megaw, 1973; Donnay \& Le Page, 1978) have called attention to existing confusion in descriptions of the quartz structure. For instance, Lang (1965) and Barron et al. (1976) gave recommendations which are correct but not wholly explicit. Megaw (1973) described the structure more fully in her book Crystal Structures: a Working Approach, but unfortunately she also made a mistake in describing the quartz structure.

When I pointed this out to her, she thought it would be timely for us to write together a short paper explaining how to describe the quartz structure correctly. When we had got as far as composing the structural diagrams and the writing of a couple of paragraphs, a definitive paper by Donnay \& Le Page (1978) appeared, explaining in considerable detail the correct way to address the quartz structure problem. They too had spotted the error in Helen's book. Helen then became discouraged and felt that there was no point in continuing with the work. As Donnay and Le Page pointed out, Megaw had intended to show the right-handed quartz structure according to the recommendation of Lang [denoted $r(-)$ by Donnay and Le Page]. But in fact, her diagram for right-handed quartz in space group $P 3_{2} 21$ is the $z(+)$ view in Fig. 1 of Donnay \& Le Page (1978).

Recently, a paper appeared entitled the Correct interpretation of diffraction properties of quartz crystals for $X$-ray optics applications (Huang et al., 2018a). Interestingly, despite the authors' reference to the Donnay and Le Page paper, they still managed to make a subtle error in their description (Huang et al., 2018b). In particular, Huang et al. (2018a) talked about 'left-handed and right-handed structures with space groups $P 3_{2} 21$ and $P 3_{1} 21$, respectively'. But what is meant by a right- 
or left-handed structure? The screw axes may be left and right handed, but that is not the same as the handedness of the structure. When one talks about left and right quartz one refers to its morphology and sense of optical rotation as viewed down the [001] direction. More importantly, Huang et al. (2018a) also considered a unit cell in which the Si coordinates are at $(u, 0,0),(-u,-u, 1 / 3)$ and $(0, u, 2 / 3)$, which they correctly described as forming a left-handed helix (LS), ${ }^{1}$ but then they stated that the handedness of this helix is the same as the 'optical handedness' (i.e. the direction the quartz crystal rotates the plane of polarized light). Now this means that their choice of arrangement of $\mathrm{Si}$ atoms should give rise to laevorotation. However, the space group defined by their choice of $\mathrm{Si}$ positions is of type $P 3_{2} 21$, which in fact corresponds to dextrorotatory or right quartz. They went on to state that their choice of structure corresponds to the $z(+)$ setting of left-handed quartz in the right-handed coordinate system of Donnay and Le Page. This is incorrect, as their setting corresponds to the $z(+)$ setting of Fig. 1.7 of Donnay \& Le Page in a right-handed coordinate system which is for righthanded quartz.

The connection between optical rotation and structure was first explained in the article by Glazer \& Stadnicka (1986), where it was shown why it is that in quartz the space group which has an RS helical arrangement of $\mathrm{Si}, P 3_{1} 21$, corresponds to laevorotation, whereas the one with an LS helical arrangement, $P 3_{2} 21$, corresponds to dextrorotation. As explained there, part of the reason for confusion lies in the fact that conventionally, when we describe the hand of a helix, we refer to the way it turns on going away from the observer, whereas in describing optical rotation, the sense of rotation of the light is described for light coming towards the observer. The fact that much of the literature was confused about these conventions led Glazer and Stadnicka to formulate an explanation of the connection between optical rotation and the senses of structural helices. Following this, a full theoretical derivation was given by Devarajan \& Glazer (1986). Note that an RS helix turns to the right on going away from the observer and an LS helix turns the opposite way. Thus, if it is the case that the light rotates in the same sense as a structural helix then an LS structural helix corresponds to clockwise (righthanded) optical rotation, and vice versa. In addition, there is a question over which helix one refers to in a particular structure. In quartz, in fact, there are two of opposing hand: one that is sixfold (although slightly distorted in low quartz) and another type that is threefold. Another point of confusion with regard to quartz in particular is that crystal growers when referring to left and right quartz (meaning its optical rotation and crystal morphology) tend to use left- and right-handed coordinate systems, respectively, whereas crystallographers

\footnotetext{
1 The abbreviations LS and RS usually mean left-handed screw and righthanded screw, as used by Donnay and Le Page. However, the use of the word 'screw' can be misleading because structural helices may not be directly related to an actual screw axis. For example, in low quartz one finds around the origin a slightly distorted sixfold helix with a threefold screw axis at the centre. For this reason, in this paper LS and RS simply mean left-handed and righthanded helices. Other examples can be found in the article by Glazer \& Stadnicka (1986).
}

normally use only a right-handed set of axes for both hands. This is well addressed by Donnay and Le Page.

Given that errors still seem to occur, it occurred to me that it would be worth reviving our original paper, in which I think the description is simpler to follow than that given by Donnay and Le Page. As Helen Megaw died 12 years ago, I felt that this paper would best honour her contribution by dedicating it to her memory.

\section{Handedness}

This is an intrinsic property of the crystal we choose to study, recognized either from its morphology or its sense of rotation of the plane of polarization of light. Both definitions agree. Note that there is a phase transition in quartz at around $846 \mathrm{~K}$ (at ambient pressure) where the space group changes, despite the fact that the handedness does not.

'Structural handedness' is a term open to misunderstanding. To what structural feature do we refer as a criterion? Consider Fig. 1(a), representing the arrangement of $\mathrm{Si}$ atoms in righthanded high quartz. Its space group is of type $P 6_{2} 22$. Note that the $6_{2}$ axis creates an RS helix around the origin, as indicated
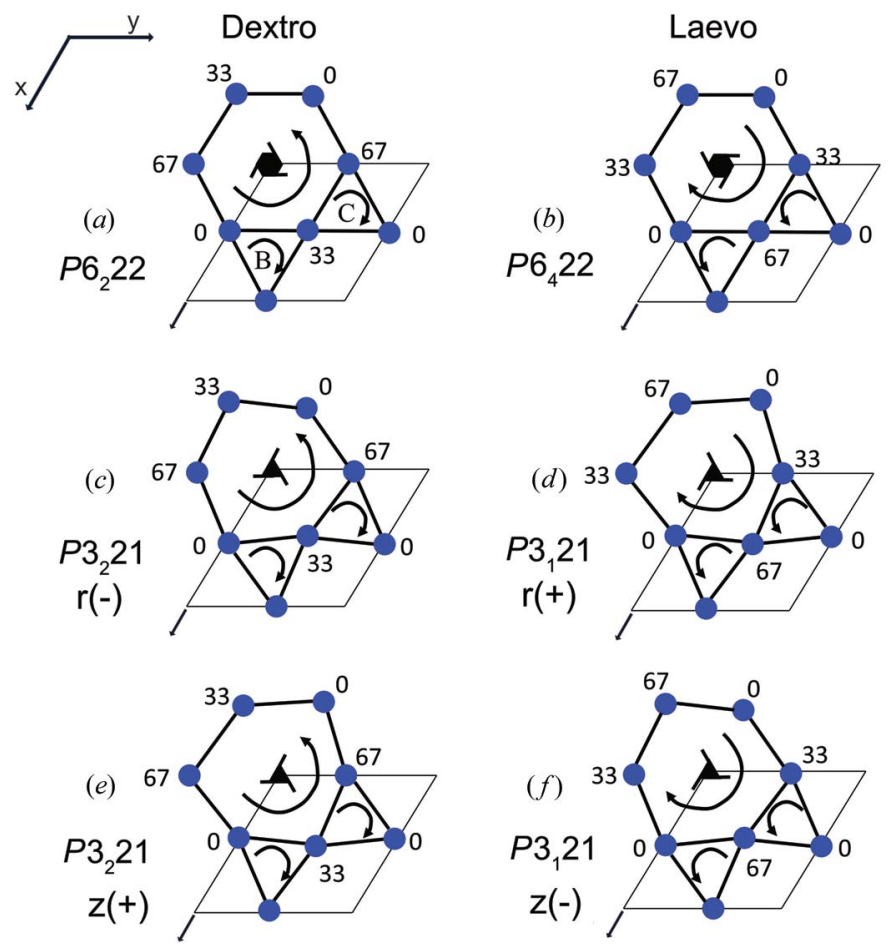

Figure 1

Arrangement of $\mathrm{Si}$ atoms in high and low quartz in different settings, using a right-hand coordinate system of axes. Heights are given as fractions of 100. In all cases the reference diad axis, as marked by an arrow, is chosen to be along $x, 0,0$. The curved arrows indicate the direction in which the helices of $\mathrm{Si}$ atoms increase in height towards the observer. $(a),(c)$ and $(e)$ are for right (dextro) quartz, while $(b),(d)$ and $(f)$ are for left (laevo) quartz. $(a)$ and $(b)$ are for high quartz in space groups of type $P 6_{2} 22$ and $P 6_{4} 22$, respectively. (c) and (d) are in space groups of type $P 3_{2} 21$ and $P 3_{1} 21$, respectively, in the obverse setting. (e) and $(f)$ are again in space groups of type $P 3_{2} 21$ and $P 3_{1} 31$, respectively, but in the reverse setting. $r(+), r(-), z(+)$ and $z(-)$ refer to the settings shown in Fig. 1 of Donnay \& Le Page (1978). 
by the curved arrow. However, there are also tight threemembered LS helices (they turn to the left away from the observer) surrounding the $3_{2}$ axes at $\mathrm{B}$ and $\mathrm{C}$, which are associated with the right turning of rotation of the plane of polarization of light (Wooster, 1953; Glazer \& Stadnicka, 1986). It is because of this that it was originally said 'The structural handedness is opposite to the morphological'. Against this, the unwary newcomer more readily notices the wider double-stranded six-membered helices around the origin, which are RS: if this type of helix correlated directly with the sense of optical rotation then we would conclude, incorrectly, that it was laevorotatory. (Incidentally, Glazer and Stadnicka showed that in $\mathrm{HgS}$ the arrangement of anisotropic polarizabilities in the equivalent sixfold helix of $\mathrm{S}$ atoms actually makes a strong dextrarotatory contribution to the overall optical rotation.) Thus statements such as 'Interestingly, the polarization planes rotate in the same direction, either left or right, as the $\mathrm{SiO}_{4}$ chains' (Akhavan, 2015) are incorrect or at best misleading. As Huang et al. (2018a) referred to Akhavan in their paper, this may be why they were misled. The same facts are true for the two settings of righthanded low quartz shown in Figs. 1(c) and 1(e).

Some writers, however, appear to associate 'structural handedness' with the type of screw axes of the space group. The fallacy of this can be seen by comparing Fig. 1(a) with Fig. $1(c)$ or $1(e)$. The screw axis changes from $6_{2}$ (RS screw axis) to $3_{2}$ (LS screw axis) at the transition, without any change of handedness of the structural helices or the physical properties associated with them. For left-handed (laevo) quartz (Figs. $1 b, 1 d$ and $1 f$ ) the screw axes are $6_{4}(\mathrm{LS})$ and $3_{1}$ (RS). A wrong association of $6_{2}$ with $3_{1}$ for left-handed quartz was made a long time ago by Wyckoff (1922). To be clear, in quartz, the sense of rotation of light corresponds to the senses of the small tight three-membered helices, as explained by Glazer \& Stadnicka (1986).

\section{Setting}

The conventional choice of axes, defined by the morphology, uses the obverse setting. Rotation of the crystals through $180^{\circ}$ about [001], but keeping the axes fixed, gives the reverse setting. Since the latter is a physically different aspect, all planes with conventional indices $(h k . l)$ will now appear as $(\overline{h k} . l)$. To avoid the consequent confusion, it is generally agreed that the obverse setting is taken as the standard. However, many X-ray workers unfortunately use the reverse setting: detailed lists are given by Lang (1965), Barron et al. (1976) and Donnay \& Le Page (1978). To check the setting, intensities can be calculated from the atomic positions: with the obverse setting $10 \overline{1} 1$ is stronger than $10 \overline{11}$, and $30 \overline{3} 1$ is much stronger than $30 \overline{3}$.

Figs. 1(c) and 1(e) show the obverse and reverse settings for right quartz, and Figs. $1(d)$ and $1(f)$ those for left quartz. Rotation of Figs. $1(c)$ and $1(d)$ through $180^{\circ}$ about [001], but keeping the axes fixed, gives Figs. 1(e) and 1(f), respectively.

It is sometimes more helpful to derive the reverse setting from the obverse by allowing small displacements of atoms.
The atoms are pictured as moving along lines joining each to its high-quartz special position and continuing beyond it to a point equidistant on the opposite side. For $\mathrm{Si}$ atoms, the displacements are along the diad axes [compare Figs. 1(c) through 1(a) with Fig. 1(e), or Figs. 1(d) through 1(b) with Fig. $1(f)]$. The mechanism of processes occurring near the high-temperature phase transition can thus be described either by keeping the axes fixed in space and allowing the displacements from the high-quartz positions to change sign, or by rotating the axes, when necessary, through $180^{\circ}$ so that the displacements always have the sign (arbitrarily called positive) corresponding to an obverse setting. For Dauphiné twinning, for example, which results from the occurrence of displacements of opposite sign (relative to fixed axes) in different regions of the crystal, rotated axes are generally used to describe the twin component whose setting would otherwise be reverse. For other processes, however, dynamic processes, in particular rotating the axes of reference as the atomic positions changed, would be impossibly confusing; here the reverse setting must be allowed.

Accepting the rule that the obverse setting should be taken as standard, we admit the qualification that the use of the reverse setting is also acceptable if, without it, a process could not be described using axes fixed in space. Care must be taken then to ensure that any unconventional use of indices is not misunderstood. $^{2}$

\section{Choice of origin}

In space groups of type $P 3_{2} 21$ there are three possible choices of origin, giving different heights for the diad axis parallel to the $x$ axis. Various authors, including Megaw (1973), chose it so as to put a diad at $(x, 0,0)$; however, in International Tables for Crystallography, Vol. A (ITA), the diad is at $(x, 0,1 / 6)$. On the other hand, for the space groups of type $P 6_{2} 22$, which have subgroups of type $P 3_{2} 21$, ITA's choice of origin puts a diad at $(x, 0,0)$. As pointed out by Megaw in her book (see Figs. 11-20 therein), since the Si atom lies on the diad, to follow ITA's choice for both space groups would mean making a discontinuous change of $z=1 / 3$ in the Si coordinate at the transition (with a corresponding change for the $\mathrm{O}$ atoms) - a very confusing requirement for the description of a near-continuous transition. There is a similar incompatibility in ITA's choices for space groups of type $P 3_{1} 21$ and $P 6_{4} 22$.

It is simpler to put the prototype $\mathrm{Si}$ at height 0 rather than $1 / 3$, and this was the earlier usage - retaining ITA's origin for high quartz. This is the choice of origin used in Fig. 1 and is implicit in the parameters used by Barron et al. (1976) and Donnay \& Le Page (1978). The combination of ITA's origin

\footnotetext{
2 The reverse setting seems to be widely used by the X-ray community (see for example the American Mineralogist database, http://rruff.geo.arizona.edu/ AMS/amcsd.php, and search for quartz), whereas quartz crystal suppliers normally deliver crystal wafers in the obverse setting. This affects one's choice of reflection indices. It is important, therefore, to recognize this difference or better still to fix on one convention for both cases. The rhombohedral space group types given by International Tables for Crystallography, Vol. A, are in the obverse setting and so I suggest (and Megaw did too) that this should be preferred for the structural descriptions as well.
} 
for low quartz with an origin for high quartz displaced by $z=$ $1 / 3$ from that of ITA would be an acceptable alternative.

\section{Standard description}

Consider right-handed (dextrorotatory) low quartz in the obverse setting. The space group is of type $P 3_{2} 21$ (Fig. $1 c$ ), corresponding to the $r(-)$ setting of Donnay \& Le Page (1978). Right-handed axes of coordinates are of course used.

The atomic coordinates are

$$
\begin{array}{lllll}
3 a & \mathrm{Si} & x, 0,0 & 0, x, 2 / 3 & -x,-x, 1 / 3 \\
6 c & \mathrm{O} & \begin{array}{l}
\text { (1) } x, y, z \\
\text { (4) } y, x, 2 / 3-z
\end{array} & \text { (2) }-y, x-y, 2 / 3+z & \text { (3) } y-x,-x, 1 / 3+z \\
& & \text { (5) } x-y,-z & \text { (6) }-x, y-x, 1 / 3-z
\end{array}
$$

As the space group of low quartz is a maximal non-isomorphic subgroup of that of high quartz $\left(P 3_{2} 21\right.$ versus $P 6_{2} 22$ with basis vectors in common, apart from the $z / 3$ shift of the origin already mentioned), the coordinates $1-6$ are simply obtained by selecting coordinate positions $1,2,3,7,8$ and 9 from $P 6_{2} 22$ of ITA.

Typical parameter values are

for Si, $x=0.535$; for O, $x=0.415, y=0.143, z=0.213$.

Note that in Fig. 1 only the Si atoms are shown. The prototype $\mathrm{Si}$ atom is at $(x, 0,0)$. This lies further from the origin of the unit cell than its equivalent atom at $(-x, 0,0)$, and corresponds to the direction in which a negative piezoelectric charge is created on extension. As pointed out by Lang (1965) the unit-cell axes point along the directions from the centre of the distorted hexagon of $\mathrm{Si}$ atoms around the origin of the unit cell towards the acute angle. Fig. 1(c) is equivalent to the Fig. $1.5 r(-)$ setting of Donnay and Le Page (although shown rotated through $120^{\circ}$ about [001]).

The relationship to high quartz can be shown by writing the parameters in terms of a constant quantity $d$ and difference parameters $s, u$ and $w$ :

for $\mathrm{Si}, x=1 / 2+s$; for $\mathrm{O}, x=2 d, y=d-u, z=1 / 6+w$.
The numerical values are

$$
s=0.035, d=0.207, u=0.064, w=0.046 .
$$

For high quartz, $d$ is almost unchanged, while $s, u$ and $w$ become zero. The space group is of type $P 6_{2} 22$, with atomic coordinates as follows (as listed in ITA):

$$
\begin{array}{ccllc}
3 c & \mathrm{Si} & 1 / 2,0,0 & 0,1 / 2,2 / 3 & 1 / 2,1 / 2,1 / 3 \\
6 j & \mathrm{O} & 2 x, x, 1 / 6 & -x, x, 5 / 6 & -x,-2 x, 1 / 2 \\
& & x, 2 x, 1 / 2 & -2 x,-x, 1 / 6 & x,-x, 5 / 6
\end{array}
$$

where $x \simeq d=0.20$

\section{Acknowledgements}

I am most grateful to Professor Massimo Nespolo of the University of Lorraine for correcting my own errors made initially in the writing of this paper. I am also grateful to Professor Xian-Rong Huang (Advanced Photon Source, Argonne National Laboratory) for pointing out to me the disconnect between the use of obverse and reverse settings between many X-ray crystallographers and quartz crystal suppliers, and for helpful comments.

\section{References}

Akhavan, A. C. (2015). The Quartz Page, http://www.quartzpage.de.

Barron, T. H. K., Huang, C. C. \& Pasternak, A. (1976). J. Phys. C Solid State Phys. 9, 3925-3940.

Devarajan, V. \& Glazer, A. M. (1986). Acta Cryst. A42, 560-569.

Donnay, J. D. H. \& Le Page, Y. (1978). Acta Cryst. A34, 584-594.

Glazer, A. M. \& Stadnicka, K. (1986). J. Appl. Cryst. 19, 108-122.

Huang, X.-R., Gog, T., Kim, J., Kasman, E., Said, A. H., Casa, D. M., Wieczorek, M., Hönnicke, M. G. \& Assoufid, L. (2018a). J. Appl. Cryst. 51, 140-147.

Huang, X.-R., Gog, T., Kim, J., Kasman, E., Said, A. H., Casa, D. M., Wieczorek, M., Hönnicke, M. G. \& Assoufid, L. (2018b). J. Appl. Cryst. 51, 966-967.

Lang, A. R. (1965). Acta Cryst. 19, 290-291.

Megaw, H. D. (1973). Crystal Structures: a Working Approach. London: Saunders.

Wooster, W. A. (1953). Rep. Prog. Phys. 16, 62-82.

Wyckoff, R. W. G. (1922). The Analytical Expression of the Results of the Theory of Space-Groups. Carnegie Institution of Washington, Publication No. 318. Washington, DC: The Technical Press. 